\title{
Outcomes of Silicone Oil Removal in Complex Retinal Detachment
}

\author{
Ritesh Shah ${ }^{1}$, Raghunandan Byanju ${ }^{1}$, Sangita Pradhan ${ }^{1}$ \\ ${ }^{1}$ Bharatpur Eye Hospital, Bharatpur
}

\begin{abstract}
Introduction: Pars plana vitrectomy in combination with intraocular tamponade with silicone oil is a standard technique in the treatment of complex retinal detachment. Although the use of silicone oil has improved the results of retinal detachment surgery, its removal is recommended due to the ocular complications related to its long term use. However, retinal redetachment can occur after silicone oil removal.

Objective: To evaluate the anatomical and visual outcome after silicone oil removal (SOR) in eyes with complicated retinal detachment.

Material and Methods: We retrospectively analyzed 64 eyes of 64 consecutive patients of silicone oil removal over a period of 12 months. All eyes had undergone standard 3 ports pars plana vitrectomy with silicone oil placement for complicated retinal detachment. Cases that completed at least 1 month follow up duration after SOR were included in the study.

Anatomical success after SOR was achieved in 56 of 64 eyes (87.5\%). Seven of 8 redetachments $(87.5 \%)$ were seen in eyes with silicone oil tamponade duration of less than 6 months. Visual acuity improved or was stabilized in 49 of 64 eyes (76.6\%). Using paired T-test, it was found that there was no significant difference in pre and post SOR visual acuity. Postoperative ocular hypertension, corneal decompensation, band shaped keratopathy and hypotony was observed in $9.4 \%, 4.7 \%, 6.4 \%$ and $21.9 \%$ respectively.
\end{abstract}

Conclusion: Although there was no significant improvement in visual acuity, redetachment and complication rates were comparable to other studies. The duration of endotamponade was not significantly associated with the redetachment rate.

Key words: Silicone oil removal, Redetachment, Visual outcome, Keratopathy, ocular hypertension.

\section{Introduction}

Silicone oil has been used for endotamponade in complex retinal detachment with encouraging results for over four decades. Though silicone oil use was first considered in retinal surgery by

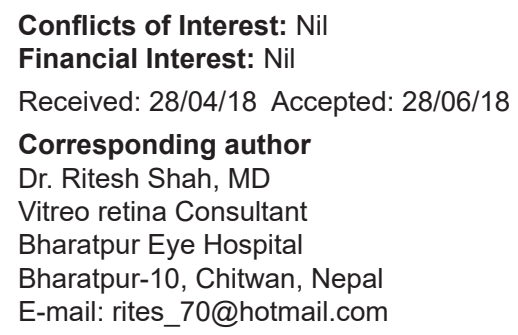

Cibis (1962), Scott (1977) modified and refined its use. In modern vitreoretinal surgery, silicone oil use is a standard practice in complicated retinal detachment to improve visual prognosis. However, long term ocular complications of silicone oil, particularly keratopathy, glaucoma and cataract, mandate its removal as soon as retina appears stable. Nevertheless, removal of silicone oil can cause reproliferation of epiretinal membranes leading to redetachment. In this study, we analyzed the records of 64 
patients to evaluate the visual and anatomical outcome along with the incidence of silicone oil related complications after silicone oil removal (SOR).

\section{Material and methods}

We performed a retrospective review of records of 64 eyes of 64 consecutive patients who underwent silicone oil removal over one year, between January 2017 and December 2017 at Bharatpur eye hospital, Bharatpur, Nepal. The research was approved by IRC of Bharatpur eye hospital. Subjects who had not completed one month follow up period were excluded from the study.

The initial surgical procedure included 360-degree scleral buckle with 240 silicone band, 20-gauge pars plana vitrectomy (Faros, Oertli, Switzerland), dissection of epiretinal membrane, laser endophotocoagulation and infusion of 1000-centistoke silicone oil (Aurosil, Aurolab, India) into the vitreous cavity.

Criteria for silicone oil removal was a stable and attached retina posterior to the encircling scleral buckle without any traction on the retina for at least 3 months.

Silicone oil was removed through two corneoscleral incisions in cases of aphakia, or through two pars plana sclerotomies in phakic and pseudophakic patients. When additional procedures such as removal of epiretinal membranes had to be carried out, a third-port was used.

Data regarding age, gender, indication for silicone oil use, duration of tamponade, followup time was recorded. Ocular examination findings before and after silicone oil removal were recorded. These examinations included best corrected visual acuity testing using a Snellen eye chart, measurement of intraocular pressure (IOP), slit lamp biomicroscopy and funduscopy with $90 \mathrm{D}$ (dioptre) and $20 \mathrm{D}$ lens.
Anatomical success was defined as a completely attached retina until the last follow-up. A visual acuity variation was considered improved or deteriorated if the difference between baseline and final BCVA was $0.2 \log$ MAR.

SPSS version 20.0 was used to perform statistical analyses. For statistical purposes visual acuity was converted to logarithm of the minimum angle of resolution ( $\log M A R)$. A modified scale was used to assign nonnumerical visual acuities to $\log$ MAR scores of 1.7, 2.0, 2.3, and 3.0 for "counting fingers", "hand movements", "perception of light", and "no perception of light" respectively (Iverson et al., 1990) .

Paired T test was used to compare preoperative and postoperative visual acuities. Univariate analysis was performed with the Pearson chi-square exact test to determine if the preoperative clinical variables were associated with the postoperative anatomical outcome.

\section{Results}

\section{Study group}

Sixty four eyes of 64 patients were included in this study. The series included 53 male patients $(82.8 \%)$ and 11 female patients $(17.2 \%)$. The mean age of the patients was 41.2 years (SD $\pm 18.8)$.

The most common indication for silicone oil use was proliferative vitreoretinopathy $(59.4 \%)$ followed by ocular trauma (18.8\%). (Table 1).

The mean duration of tamponade was 8.2 months ( $\mathrm{SD} \pm 10.8)$. Eyes with tamponade duration of more than 1 year were mostly cases operated at other centres $(6.25 \%)$. Forty-one (64\%) eyes underwent SOR within 6 months of tamponade. The mean follow up time was 2.9 months $(\mathrm{SD} \pm 3)$.

\section{Anatomical success}

Anatomical success was achieved in 56 of 64 eyes $(87.5 \%)$ with a single surgery. Retinal 
redetachment was seen in 5 of 38 eyes (13.2\%) with proliferative vitreoretinopathy, 1 of 12 eyes $(8.3 \%)$ with ocular trauma, 1 of 6 eyes $(16.6 \%)$ with proliferative diabetic retinopathy and 1 of 4 eyes $(25 \%)$ with choroidal coloboma. All these eyes had an anatomical failure within two months of silicone oil removal and resulted from either new break or reproliferation of membranes. The mean duration of redetachment was 23 days. Six of 8 eyes $(75 \%)$ had redetachment within the first 3 weeks of SOR. All the cases of redetachment underwent vitrectomy with repeat silicone oil injection. The mean duration of tamponade in cases with redetachment was 4.25 months (Range $=2.5$ to 6.5 months). Although statistically insignificant, most $(87.5 \%)$ redetachments were observed in eyes with tamponade duration of less than 6 months. $(p=.118)$

\section{Visual acuity}

Mean best corrected visual acuity preoperatively (BCVA) (1.41 log MAR) and postoperatively at 1 month (1.51) and final follow up (1.51) has been presented in Table 2. BCVA following removal of silicone oil (table 2) improved in 13 eyes $(20.3 \%)$, remained unchanged in 36 eyes $(56.3 \%)$ and worsened in 15 eyes $(23.4 \%)$ 1 month after silicone oil removal. (Table 3) The deterioration in visual acuity was due to redetachment in $8 / 15$ eyes $(38.1 \%)$. Other causes were hyphema (25\%), keratopathy $(12.5 \%)$, blood in vitreous cavity $(12.5 \%)$, optic atrophy (12.5\%), presence of perfluoropropane gas in vitreous cavity (12.5\%), and unknown cause $(12.5 \%)$.

\section{Complications}

Cataract formation was seen in 7 of 8 phakic patients and 4 eyes underwent cataract extraction with intraocular lens implantation. At the time of silicone oil removal 38 of 64 eyes were aphakic and only one eye underwent secondary IOL implantation in sulcus during SOR. The remaining aphakic eyes were planned for secondary IOL implantation only if the retina remained attached for three months post SOR and there was a significant improvement in visual acuity with aphakic correction.

Postoperative keratopathy with corneal decompensation was observed in 3 eyes (4.7\%). and all the cases were aphakic.

Preoperatively elevated IOP was observed in 6 eyes (9.4\%). Mean duration of tamponade in eyes with increased IOP was 19.25 months. Postoperatively, IOP was normalized in 2 eyes whereas it remained to be high in 4 eyes. Two eyes with preoperative normal IOP developed elevated IOP after removal of silicone oil. All except one had their IOP controlled with antiglaucoma medications. One eye with uncontrolled IOP despite undergoing trabeculectomy was referred to another centre. Three eyes had pale disc and were associated with increased IOP preoperatively. Neovascularisation of iris (NVI) was seen in one eye with high IOP.

Table 1: Indications of silicone oil use.

\begin{tabular}{|l|l|l|}
\hline Indications & Number & Percentage \\
\hline Proliferative vitreoretinopathy & 38 & 59.4 \\
\hline Ocular trauma & 12 & 18.8 \\
\hline Proliferative diabetic retinopathy & 6 & 9.4 \\
\hline RD with Choroidal coloboma & 4 & 6.3 \\
\hline TRD secondary to BRVO & 4 & 6.3 \\
\hline
\end{tabular}


Table 2: Pre and post SOR BCVA.

\begin{tabular}{|l|l|l|l|l|}
\hline & Maximum & Minimum & Mean & P value \\
\hline Pre SOR BCVA (log MAR) & 0.3 & 3 & $1.41 \pm 0.49$ & \multirow{2}{*}{0.092} \\
\hline Post SOR BCVA at 1 month (log MAR) & 0.17 & 3 & $1.51 \pm 0.59$ & 0.09 \\
\hline Post SOR BCVA at final follow up (log MAR) & 0.17 & 3 & $1.51 \pm 0.6$ & \\
\hline
\end{tabular}

Table 3: Change in BCVA after SOR.

\begin{tabular}{|l|l|l|}
\hline BCVA change & Post SOR at 1 month & Post SOR at final follow up \\
\hline Improved & 13 & 14 \\
\hline No change & 36 & 34 \\
\hline Worse & 15 & 16 \\
\hline
\end{tabular}

Other anterior segment complications like hypotony and band shaped keratopathy were observed in 14 eyes $(21.9 \%)$ and 4 eyes $(6.4 \%)$ respectively.

\section{Discussion}

In this case series, the rate of redetachment was $12.5 \%$. The reported rate of redetachment after silicone oil removal varies from 9 to $25 \%$. (La Heij et al., 2001; Van Meurs et al., 1993; Bassat et al., 2000; Jonas et al.,2001; Falkner et al., 2001; Unlu et al., 2004; Casswell et al.,1987; Jiang et al., 2002; Zilis et al., 1989; Franks et al., 1991; Eckardt et al., 1992) This variation can be accounted to different follow up period in different studies. The redetachment rate in this study was in the lower range compared to the above studies. However, this needs to be confirmed with a longer follow up period. Interestingly in this study, the duration of tamponade had no significant effect on anatomical outcome. However, most redetachments $(87.5 \%)$ were observed in eyes with duration of tamponade less than 6 months. This was similar to many other studies who also observed no effect of tamponade duration on redetachment. (Falkner et al., 2001; Goezinne et al., 2007; Fathalla et al.,2015)

A favorable visual outcome was seen in this study although the difference in mean best corrected pre- and post SOR visual acuity was not statistically significant $(p=0.092)$. Visual acuity was stabilised in more than half of the cases $(56.3 \%)$ and almost equal number of eyes had either improvement or deterioration of visual acuity. In contrast, many other studies have reported improvement of visual acuity in significant number of eyes (Falkner et al., 2001; Goezinne et al., 2007; Tavares et al., 2015). Pre-SOR poor visual acuity of counting finger or worse in 30 eyes $(46.8 \%)$ and hand motion or worse in fifteen eyes $(23.3 \%)$ can probably explain the relatively less improvement in visual acuity following SOR in this study.

Keratopathy associated with corneal decompensation was seen in $4.7 \%$. Other studies have reported rate of keratopathy ranging from $4.5 \%$ to $100 \%$. (Falkner et al., 2001; Federman et al., 1988; Abrams et al., 1995; Franks et al., 1991; Yeo et al.,1987). This variation may be the result of different definitions used in various studies. However in this study only permanent keratopathy with corneal decompensation was considered whereas Silicone Study Report no. 6 (Hutton et al., 1994) included band shaped keratopathy, corneal decompensation in the same category. Band shaped keratopathy in this study was seen in 4 eyes $(6.4 \%)$. Superficial scraping was done in 2 cases where central cornea was involved although a proper surgical debridement with a neutral solution of disodium ethylenediaminetetraacetic acid (EDTA) or phototherapeutic keratectomy (PTK) with excimer laser is recommended in treatment of 


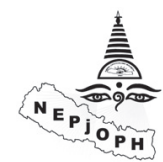

BSK. (Sharma N et al., 2011; Najjar DM et al., 2004)

Postoperative ocular hypertension $(9.4 \%)$ was comparable to other studies (Falkner et al., 2001; Goezinne et al., 2007). The mean duration of tamponade in eyes with ocular hypertension (19.2 months) was remarkably high compared to mean tamponade duration of the entire study group (8.2 months). However, no conclusion could be drawn regarding the association of duration of tamponade and raised IOP probably due to small sample size.

Regarding cataract formation, 7 of 8 phakic eyes developed cataract. Since the number of phakic eyes was very less, the findings are also inconclusive.

\section{Conclusion}

In this series, no significant change in pre and post SOR visual outcome was seen. However, visual status was stabilized in most of the cases and redetachment rate was relatively low. Thus we conclude that silicone oil removal after a thorough follow up evaluation can result in stabilization of retina and visual acuity in most eyes.

\section{References}

Cibis PA, Becker B, Okun E, Cannan $S$ (1962). The use of liquid silicone in retinal detachment surgery. Arch Ophthalmol; 68:5909.

Scott JD (1977). A rationale for the use of liquid silicone. Trans Ophthalmol Soc UK ; 97:235-7.

Iverson DA, Ward TG, Blumenkranz MS (1990). Indications and results of relaxing retinotomy. Ophthalmology; 97:1298-1304.

La Heij EC, Hendrikse F, Kessels AG (2001). Results and complications of temporary silicone oil tamponade in patients with complicated retinal detachments. Retina; 21: 107-14.
Shah R et al Outcome of Silicon Oil Nepal J Ophthalmol 2018; Vol 10 (20): 124-129

Van Meurs JC, Mertens DA, Peperkamp E, Post J (1993). Five year results of vitrectomy and silicone oil in patients with proliferative vitreoretinopathy. Retina; 13: 285-9.

Bassat IB, Desatnik H, Alhalel A, Treister G, Moisseiev J (2000). Reduced rate of retinal detachment following silicone oil removal. Retina; 20: 597-603

Jonas JB, Knorr HL, Rank RM, Budde WM (2001). Retinal redetachment after removal of intraocular silicone oil tamponade Br J Ophthalmol; 85: 1203-7.

Falkner CI, Binder S, Kruger A (2001). Outcome after silicone oil removal. $\mathrm{Br} \mathrm{J}$ Ophthalmol; 85: 1324-7.

Unlu N, Kocaoglan H, Acar MA, Sargin M, Aslan BS, Duman S (2004). Outcome of complex retinal detachment surgery after silicone oil removal. Int Ophthalmol; 25: 33-6.

Casswell AG, Gregor ZJ (1987). Silicone oil removal. II. Operative and postoperative complications. Br J Ophthalmol; 71: 898-902.

Jiang F, Krause M, Ruprecht KW, Hille K (2002). Risk factors for anatomical success and visual outcome in patients undergoing silicone oil removal. Eur J Ophthalmol; 12: 293-8.

Zilis JD, McCuen BW, de Juan E, Jr., Stefansson E, Machemer R (1989). Results of silicone oil removal in advanced proliferative vitreoretinopathy. Am J Ophthalmol; 108: 1521.

Franks WA, Leaver PK (1991). Removal of silicone oil-rewards and penalties. Eye; 5(3): 333-7.

Eckardt C, Behrendt S, Zwick A (1992). Results of silicone oil removal from eyes treated with retinectomies. Ger J Ophthalmol; 1: $2-6$.

Goezinne F, La Heij EC, Berendschot TTJM, Liem ATA, and Hendrikse F (2007). 
Risk factors for redetachment and worse visual outcome after silicone oil removal in eyes with complicated retinal detachment European Journal of Ophthalmology; 17: 627-637.

Fathalla AM, Mohamed TA, Al-Sebaity DM (2015). Early versus late silicone oil removal after pars plana vitrectomy for rhegmatogenous retinal detachment in Upper Egypt. Egypt Retina J; 3:39-44.

Tavares R, Nobrega MJ, Nobrega F, Novelli F, Oliveria C (2015). Timing and outcomes after silicone oil removal in proliferative vitreoretinopathy: a retrospective clinical series. International Journal of Retina and Vitreous; 1:2. DOI 10.1186/s40942-0150002-y.

Federman JL, Schubert HD (1988). Complications associated with the use of silicone oil in 150 eyes after retina-vitreous surgery. Ophthalmology; 95: 870-6.

Abrams GW, Azen SP, McCuen BW, Flynn HW Jr, Lai MY, Ryan SJ (1995). The incidence of corneal abnormalities in the
Silicone Study. Silicone Study Report 7. Arch Ophthalmol; 113: 764-9.

Franks WA, Leaver PK (1991). Removal of silicone oil-rewards and penalties. Eye; 5: 333-7.

Yeo JH, Glaser BM, Michels RG (1987). Silicone oil in the treatment of complicated retinal detachments. Ophthalmology; 94: 110913.

Hutton WL, Azen SP, Blumenkranz MS, et al (1994). The effects of silicone oil removal. Silicone Study Report 6. Arch Ophthalmol; 112: 778-85.

Sharma N, Mannan R, Sinha R, Kaushal S, Titiyal JS, Kumar A, Vajpayee RB (2011). Excimer laser phototherapeutic keratectomy for the treatment of silicone oil-induced bandshaped keratopathy. Eye Contact Lens; 37:2825.

Najjar DM, Cohen EJ, Rapuano CJ, Laibson PR (2004). EDTA chelation for calcific band keratopathy: results and long-term followup. Am J Ophthalmol; 137:1056-1064. 